\title{
Telemonitoring with an implantable loop recorder in outpatient heart failure care
}

\author{
One year follow-up report from a prospective observational Dutch multicentre \\ study
}

\author{
R. S. S. Kort · Y. S. Tuininga · H. A. Bosker $\cdot$ M. Janssen $\cdot$ R. Tukkie
}

Published online: 3 December 2018

(C) The Author(s) 2018

\begin{abstract}
Introduction In the care of heart failure patients, telemonitoring is receiving growing attention. The main purpose of this study was to determine the effect of continuous telemonitoring with an implantable loop recorder (ILR, Reveal XT), a novel strategy in the management of stable heart failure patients without a cardiac implantable device. Furthermore, little is known about the incidence of subclinical arrhythmias in this specific group of patients.

Materials and Methods Stable heart failure patients, New York Heart Association Class II and III, without recent hospitalisation or upcoming intervention, were included. After implantation of the ILR there was regular contact with the research nurse on a pre-specified basis. Clinic visits and telephonic interviews were alternated for a minimum of 1 year. Parallel visits to their treating physician continued according to standard care. The treating physician was blinded for the ILR findings, accept for pre-specified, significant arrhythmic events.

Results Thirty patients were included and followed for a median duration of 12 months. In 13 patients, data from the loop recorder led to therapeutic changes.
\end{abstract}

R. S. S. Kort $(\varangle) \cdot$ M. Janssen · R. Tukkie

Department of Cardiology, Spaarne Gasthuis, Haarlem, The Netherlands

rachelkort@hotmail.com

Y. S. Tuininga

Department of Cardiology, Deventer Hospital, Deventer, The Netherlands

\section{H. A. Bosker}

Department of Cardiology, Rijnstate Hospital, Arnhem, The Netherlands
One patient received a pacemaker. Eight patients developed atrial fibrillation, all subclinical, with a mean burden of $65.8 \pm 173.2 \mathrm{~min} /$ day.

Conclusion The use of an ILR could potentially impact patient management. Additional study is needed in different patient populations (e.g. higher risk groups) to assess if an ILR could also impact on endpoints such as heart failure hospitalisation.

Keywords Heart failure - Atrial fibrillation - Telemedicine $\cdot$ Ambulatory care

\section{Introduction}

Heart failure is affecting an increasing number of people worldwide and is associated with substantial morbidity and mortality, a high risk of developing arrhythmias, hospitalisation, and thromboembolic events [1]. The occurrence of atrial fibrillation (AF) predisposes these patients to embolic events and worsening heart failure [2]. Over the last years, tele-

\section{What's new?}

- To the best of our knowledge, this study is first to report on the use of implantable loop recorders for heart failure management.

- Continuous monitoring detected arrhythmic events that led to therapeutic changes in 13 patients. These events were not detected by routine visits to the cardiologist.

- The use of an implantable loop recorder could potentially impact patient management, but additional study is needed. 
monitoring has received growing attention in different fields of medicine. Several telemonitoring entities can be distinguished ranging from telehomecare using (semi) structured telephonic assessments, via telemonitoring with transfer of physiological data (such as weight, blood pressure, and heart rate) to remote monitoring in cardiac device patients which adds data such as heart rate variability, atrial tachyarrhythmia detection, patient activity and thoracic fluid accumulation. New invasive technologies are currently being tested measuring, for example, pulmonary artery pressure $[3,4]$. These parameters are recorded with the purpose of positively influencing heart failure outcome, increasing patient participation and early adjusting therapy in case of heart failure deterioration.

Multiple studies have investigated the effect of telemonitoring on reducing hospitalisation and mortality [5-9]. Results of these studies have been contradictory. The meta-analysis by Inglis et al. suggested a positive effect of telemonitoring on all-cause mortality and hospitalisation [10]. However, these findings have recently been challenged by two large multicentre randomised trials. The Tele-HF study, using structured telephonic interviews, showed no improvement of the primary endpoint (hospital readmission within 30 days for any cause), compared with standard care [11]. Also, the TIM-HF trial requiring daily self-assessments significantly reduced all-cause mortality in only a selected group of patients with a good mental state $[8,12,13]$.

A large proportion of systolic heart failure patients receive treatment with an implantable cardioverterdefibrillator or cardiac resynchronisation therapy device (pacemaker or defibrillator). These devices offer the chance to continuously monitor physiological and technical data. In the IN-TIME trial, telemonitoring significantly reduced all-cause mortality and it was hypothesised that three mechanisms contributed in parallel to this improved clinical outcome [5]. The three proposed mechanisms were early detection of tachyarrhythmias, early recognition of suboptimal device function and raised patient awareness. In heart failure patients without a current indication for a cardiac implantable device, continuous data collection on early detection of tachyarrhythmia could also be achieved by an implantable loop recorder (ILR). No studies have been performed using an ILR for the sole purpose of early arrhythmia detection in heart failure patients. We anticipated that an ILR could be useful to fulfil this gap.

The main purpose of this study was to determine the usefulness of telemonitoring, with an ILR as a novel strategy in the management of stable heart failure patients without a cardiac implantable device and without oral anticoagulant (OAC) therapy. Secondarily, little is known about the incidence of subclinical arrhythmias in this specific group of stable heart failure patients. In this paper, we report the 1 year follow-up data.
Materials and Methods

\section{Study design}

This study was a prospective, observational multicentre study performed in three large Dutch non-academic teaching hospitals (trial registration: NCT01366703). Patients were recruited between July 2011 and April 2014 and were included after giving written informed consent. Minimal follow-up duration was 1 year. Because of the nature of the study (descriptive without statistical assumption because of lack of previous data) no formal power calculation was done to determine the sample size. A patient sample of 30 was considered reasonable. The study was approved by the Medical Research Ethics Committee of Noord-Holland.

\section{Inclusion and exclusion criteria}

Patients with stable systolic heart failure (ejection fraction $35-45 \%$ ) or diastolic heart failure (ejection fraction $>45 \%$ and echocardiographic signs of impaired diastology or NT-proBNP >250 ng/l) were included. Stable was defined as a New York Heart Association (NYHA) Class II or III, without hospitalisation in the last 3 months or a scheduled intervention in the coming 3 months. Patients with a pacemaker/ICD or an indication for such a device were excluded. Since we sought possible therapeutic implications from continuous monitoring, patients with known AF, a virtual $\mathrm{CHA}_{2} \mathrm{DS}_{2}$-VASc of 1 or less or already on an OAC were excluded.

\section{Reveal XT implantation}

After inclusion, the implantable cardiac monitor Reveal XT (Medtronic Inc., Minneapolis, USA) was inserted in a routine fashion. Patients were placed on home monitoring with Carelink and the following alerts activated: ventricular tachycardia, asystole of longer than 3 seconds, bradycardia and AF. All Carelink alerts were checked daily by a device technician during in office hours on weekdays.

\section{Follow-up}

Patients were followed in a parallel fashion. First, they were seen at the clinic by a research nurse, experienced in heart failure trials, at intake, 6 and 12 months, with additional structured telephonic interviews at month 3 and 9. The research nurse was also responsible for careful case report form (CRF) compliance and CRF documentation, collection of the pre-specified arrhythmic events in the CRF and distribution of the pre-specified arrhythmic events to the treating physician (see later). The ILR was interrogated before visiting the research nurse at month 6 and 12. Second, in parallel, patients were 
seen by their own treating physician the same day at month 6 and 12. Minimal follow-up was 1 year. Their treating physician was unaware of the ILR findings except for several pre-specified events. Pre-specified events were asystolic episodes of 3 seconds or more, $\mathrm{AF}$ or other supraventricular arrhythmia longer than 6 minutes, non-sustained ventricular tachycardia or second/third degree AV block of any duration. If a pre-specified event occurred (detected at a routine visit to the technician or by a home-monitoring alert), the device technician contacted the research nurse who then took appropriate action (CRF update and informing the treating cardiologist). Therapeutic interventions (whether based on a clinical event or after a pre-specified event from the ILR) were made at the discretion of the treating physician.

\section{Analysis}

All data were processed using IBM SPSS 20.0. Continuous data are expressed as mean \pm standard deviation, discrete data are expressed as frequency and percentage.

Data retrieved from Carelink were additionally processed at Medtronic Bakken Research (Heerlen; the Netherlands) to obtain aggregated data on arrhythmic events and AF burden, which were exported to an excel sheet. All event tracings were assessed by an experienced device cardiologist (RT).

\section{Results}

\section{Patient demographics}

Baseline characteristics are presented in Tab. 1. Between July 2011 and April 2014, 30 patients were enrolled and received an ILR. The mean age was $71.8 \pm 8.8$ years. The majority were female $(56.7 \%)$. At baseline, 25 patients were in NYHA functional class II and 5 in class III. The mean ejection fraction was $44.5 \pm 7.8$ and $80 \%$ of the patients had systolic heart failure.

\section{Follow-up}

Median follow-up was 12 months. No patients were lost to follow-up. Two ILRs were removed, at 2 and 12 months, due to local discomfort. One patient had a superficial wound infection. Three patients died during follow-up. The first patient with severe triple vessel disease and inoperable left main coronary stenosis died of acute cardiogenic shock within 1 month after ILR implantation. During this month, no events were recorded by the ILR. The second patient died due to a ruptured abdominal aortic aneurysm within 1 month after inclusion, the ILR data were lost. The last patient died in his sleep, no rhythm or conduction disorders were recorded by the
Table 1 Baseline characteristics

\begin{tabular}{l|c}
\hline age (years) & $72 \pm 8.8$ \\
\hline men & $13(43.3)$ \\
\hline weight (kg) & $78.3 \pm 16$ \\
\hline LVEF & $44.5 \pm 7.8$ \\
\hline NYHA & \\
\hline II & $25(83.3)$ \\
\hline III & $5(16.7)$ \\
\hline heart failure duration & \\
\hline$<2$ months & $4(13.3)$ \\
\hline$<3$ months & $4(13.3)$ \\
\hline$<6$ months & $2(6.7)$ \\
\hline$>6$ months & $20(66.7)$ \\
\hline medical history & \\
\hline Coronary artery disease & $11(36.7)$ \\
\hline revascularisation (PCI and/or CABG) & $9(30)$ \\
\hline valvular heart disease & $2(6.7)$ \\
\hline diabetes mellitus & $9(30)$ \\
\hline hypertension & $19(63.3)$ \\
\hline peripheral artery disease & $4(13.3)$ \\
\hline renal insufficiency (GFR <60 ml/min/1.73 m²) & $6(20)$ \\
\hline medication & \\
\hline beta blocker & $25(83.3)$ \\
\hline ACEi/ARBs & $27(90)$ \\
\hline antiplatelet therapy & $18(60)$ \\
\hline calcium antagonist & $5(16.7)$ \\
\hline antiarrhythmics & $1(3.3)$ \\
\hline diuretics & $18(60)$ \\
\hline nitrates & $4(13.3)$ \\
\hline All characteristics are reported as frequencies (percentage), & except for \\
\hline age, weight and left ventricular ejection fraction, which are reported as \\
\hline mean \pm standard deviation
\end{tabular}

ILR. No patients were admitted to the hospital for worsening heart failure.

\section{Detection of pre-specified events}

In 15 patients $(50 \%)$ pre-specified events were detected (Tab. 2). The mean time to a pre-specified event was $6.0 \pm 3.6$ months (Fig. 1). AF was recorded in 8 patients $(26.7 \%)$. ILR data led to therapeutic changes in 13 patients $(43.3 \%)$. The following therapeutic changes were implemented: pacemaker implant in 1, institution of OAC in 8 (vitamin $\mathrm{K}$ antagonists in 7, direct-acting oral anticoagulants in 1) and adjustments of beta-blocker dose in 6 patients. Of the pre-specified ILR events, none was detected by the routine clinic visits to the cardiologist. 
Table 2 Overview of detected pre-specified events and actions taken

\begin{tabular}{|c|c|c|c|c|}
\hline $\operatorname{sex}$ & age & heart failure & event & action \\
\hline female & 81 & HFREF & bradycardia/NSVT & none \\
\hline female & 68 & HFREF & asystole & dose beta blocker changed \\
\hline male & 65 & HFREF & atrial fibrillation & dose beta blocker changed/OAC started \\
\hline male & 78 & HFREF & asystole & none \\
\hline male & 86 & HFPEF & bradycardia/Atrial fibrillation & OAC started \\
\hline male & 71 & HFREF & atrial fibrillation & OAC started \\
\hline female & 77 & HFREF & SVT & dose beta blocker changed \\
\hline female & 74 & HFREF & atrial fibrillation & OAC started \\
\hline male & 59 & HFREF & atrial fibrillation & OAC started \\
\hline female & 74 & HFREF & asystole & dose beta blocker changed \\
\hline female & 65 & HFREF & atrial fibrillation & dose beta blocker changed/OAC started \\
\hline male & 62 & HFREF & atrial fibrillation & OAC started \\
\hline male & 71 & HFREF & atrial fibrillation & OAC started \\
\hline female & 71 & HFPEF & bradycardia & beta blocker stopped \\
\hline female & 80 & HFPEF & AV block & pacemaker implanted \\
\hline
\end{tabular}

\section{Atrial fibrillation detection}

In 20 patients AF was detected by the ILR, 12 were considered false-positive after expert adjudication. Therefore 8 patients were diagnosed with true $\mathrm{AF}$ of $>6 \mathrm{~min}$. By increasing the minimal duration of AF detection to $>60 \mathrm{~min}, \mathrm{AF}$ was detected in 13 patients by the ILR, 6 were labelled false-positive after expert adjudication leaving 7 patients with true $\mathrm{AF}$ of $>60 \mathrm{~min}$. The mean AF burden was $65.8 \pm 173.2 \mathrm{~min} /$ day.

\section{Discussion}

This study is the first report on ILR use, solely for telemonitoring purpose in stable moderately severe heart failure patients. Our results show that continuous monitoring of heart failure patients with an ILR detects more arrhythmic events than standard care and led to therapeutic changes in 13 patients.

The most frequently recorded pre-specified event was $\mathrm{AF}$, detected in $27 \%$ of patients, all subclinical. This percentage is in the range of previously reported prevalence of AF in heart failure [14, 15]. This finding also corresponds with the conclusion of the INTIME trial, where atrial tachyarrhythmias most often led to unplanned patient contacts [5]. Compared with the IN-TIME study, our patient population differed in several ways. First, our patients did not have a previous indication for a cardiac implantable device, second the mean ejection fraction in the IN-TIME population was much lower (mean 26\%) and third $25 \%$ of the patients in the IN-TIME trial were already known to have AF. Despite these differences, also in our patient population atrial tachyarrhythmias were prevalent, even more prevalent than might be expected for this patient cohort. There were no hospitalisations for heart failure during follow-up. This can be ex- plained by the patient population under investigation, clinically stable and with a relatively high mean ejection fraction. In further research the study population can be expanded by including less stable patients, for instance after hospital discharge for acute/worsening heart failure to judge the impact of early arrhythmia detection on heart failure hospitalisations.

The detection of subclinical AF is of clinical importance. The benefit of anticoagulant in subclinical AF is proven, but there is no consensus on the duration of AF related to the risk of ischaemic stroke. Boriani et al. demonstrated that the AF burden is an independent predictor for ischaemic stroke [16]. Of the prespecified durations (5 minutes, 1, 6, 12, and 23 hours) 1 hour was associated with the highest hazard ratio. However, Healey et al. described an already increased risk of stroke for AF duration of $\geq 6 \mathrm{~min}$ [17]. Therefore, we reported on the analysis with cut-off points of both 6 and 60 minutes. Twelve patients were labelled falsepositively as AF patients by the ILR. If corrected for $60 \mathrm{~min}$, only 6 patients remain labelled false-positive for AF. Podd et al. previously reported on the sensitivity and specificity for detection of AF of the Reveal XT at $79 \%$ and $66 \%$ respectively [18]. To strengthen the reliability of automated detection algorithms and to reduce the workload of hospital staff, the specificity of the AF detection algorithms should be improved.

\section{Limitations}

Firstly, therapy change was left to clinical judgment in our study. The institution of OACs in case of subclinical AF detected by cardiac implantable devices is under debate [19]. The impact of OAC therapy (including direct anticoagulants) in subclinical device-detected $\mathrm{AF}$ is not yet proven and currently being studied in the NOAH-AF trial (NCT02618577). 


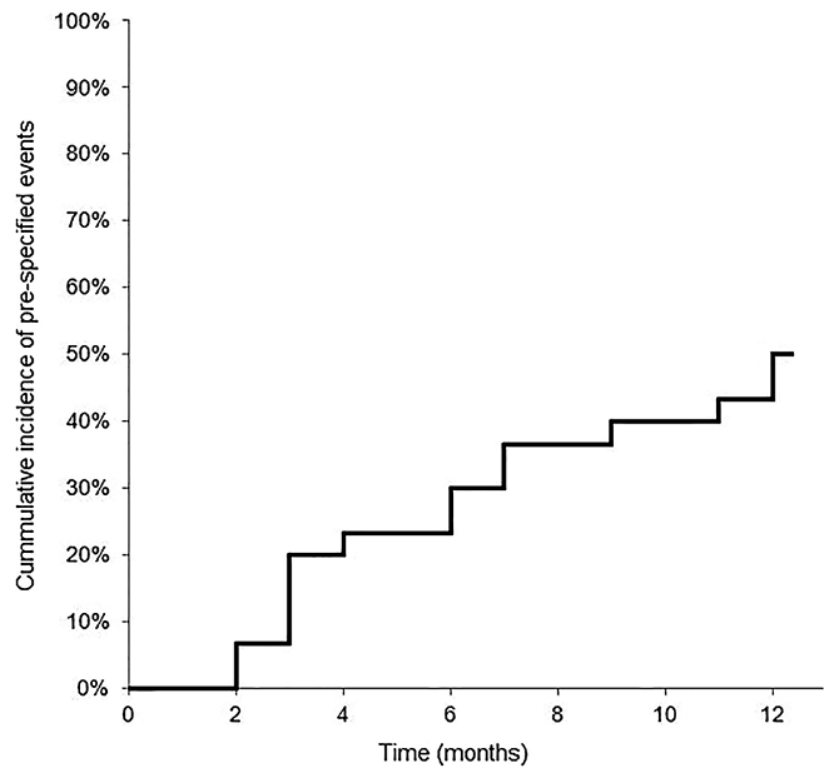

Fig. 1 Kaplan-Meier plot: Time to a pre-specified event

And second, the study was not randomised but to a certain extent the patients served as their own control group, because treating physicians were mainly blinded to the ILR findings. Although pre-specified findings were reported to the cardiologist, none of these were detected by the routine visits of standard care. Physicians might have been biased knowing that they would be provided with the ILR findings should these occur. Also, this finding does not necessarily imply that the pre-specified events would not be detected clinically later on, but telemonitoring has proven to detect clinically relevant events much earlier. In the Trust trial, the median time to evaluation was $<2$ days in the home monitoring group compared with 36 days in patients without home monitoring [20]. These findings were reproduced in the Connect trial were the median time from a clinical event to clinical decision per patient was reduced from 22 days in the in-office arm without home monitoring to 4.6 days in patient on remote monitoring [21].

Finally, only 30 patients were included over several years. No one reason can be given for this slow inclusion; however, precise data are missing since no screening log was kept. During the trial inclusion period, several competing heart failure trials were running in our departments. Also, some suitable candidates were unwilling to undergo an ILR implant.

\section{Conclusion and future implications}

We conclude that continuous monitoring of heart failure patients with an ILR detects more arrhythmic events and leads to a relevant number of therapeutic changes compared with standard care. Devices such as an ILR add considerable initial costs and a small risk of device-related complications. These risks will be reduced with the currently available small injectable ILRs. The use of an ILR could potentially impact patient management, especially when incorporating novel parameters (e.g. third heart sound detection, respiratory rate and respiratory shallowness monitoring), as recently reported in the MultiSENSE study or by combining these continuous parameters with currently available telehomecare strategies [22]. Additional randomised studies are needed in different patient populations to assess whether an ILR could also impact on cost efficiency. However, we anticipate that telemonitoring will increasingly become part of standard heart failure care.

Funding This work was supported by an unrestricted research grant from Medtronic Inc

Conflict of interest R.S.S. Kort, Y.S. Tuininga, H.A. Bosker, M. Janssen and R. Tukkie declare that they have no competing interests.

Open Access This article is distributed under the terms of the Creative Commons Attribution 4.0 International License (http://creativecommons.org/licenses/by/4.0/), which permits unrestricted use, distribution, and reproduction in any medium, provided you give appropriate credit to the original author(s) and the source, provide a link to the Creative Commons license, and indicate if changes were made.

\section{References}

1. McMurray JJV, Adamopoulos S, Anker SD, et al. ESC Guidelines for the diagnosis and treatment of acute and chronic heart failure 2012: The Task Force for the Diagnosis and Treatment of Acute and Chronic Heart Failure 2012 of the European Society of Cardiology. Eur Heart J. 2012;33:1787-847.

2. Wang TJ, Larson MG, Levy D, et al. Temporal relations of atrial fibrillation and congestive hart failure and their joint influence on morality: the Framingham Heart Study. Circulation. 2003;107:2920-5.

3. Asselbergs FW, Baars, F, Boyne, JJJ, et al. Samenwerkingsafspraken en kwaliteitscriteria bij invoeren telebegeleiding bij hartfalen in Nederland. 2016; Retrieved September 12, 2017, from http:/ /www.nvhvv.nl/userfiles / Samenwerkingsafspraken\%20en\%20kwaliteitscriteria \%20bij\%20invoeren\%20telebegeleiding.pdf

4. HasanA, PaulV.Telemonitoring in chronic heartfailure. Eur Heart J.2011;32:1457-64.

5. Hindricks G, Taborsky M, Glikson M, et al. Implant-based multiparameter telemonitoring of patients with heart failure (IN-TIME): a randomised controlled trial. Lancet. 2014;384:583-90.

6. Black JT, Romano PS, Sadeghi B, et al. A remote monitoring and telephone nurse coaching intervention to reduce readmissions among patients with heart failure: study protocol for the Better Effectiveness After Transition-Heart Failure (BEAT-HF) randomized controlled trial. Trials. 2014;15:124.

7. Chaudhry SI, Barton B, Mattera J, et al. Randomized trial of Telemonitoring to Improve Heart Failure Outcomes (TeleHF): study design. JCard Fail. 2007;13:709-14.

8. Koehler F, Winkler S, Schieber M, et al. Telemedical Interventional MonitoringinHeartFailure (TIM-HF), arandomized, controlled intervention trial investigating the impact of telemedicine on mortality in ambulatory pa- 
tients with heart failure: study design. Eur J Heart Fail. 2010;12:1354-62.

9. Sardu C, Santamaria M, Rizzo MR, et al. Telemonitoring in heart failure patients treated by cardiac resynchronisation therapy with defibrillator (CRT-D): the TELECART Study. Int JClin Pract. 2016;70:569-76.

10. Inglis SC, Clark RA, McAlister FA, Stewart S, Cleland JGF. Which components of heart failure programmes are effective? Asystematicreviewandmeta-analysis of the outcomes of structured telephone support or telemonitoring as the primary component of chronic heart failure management in 8323 patients: Abridged Cochrane review. Eur J HeartFail. 2011;13:1028-40.

11. Chaudhry SI, Mattera JA, Curtis JP, et al. Telemonitoring in patients with heart failure. NEngl J Med. 2010;363:2301-9.

12. Koehler F, WinklerS, Schieber M, etal. Telemedicinein heart failure: pre-specified and exploratory subgroup analyses from the TIM-HF trial. Int J Cardiol. 2012;161:143-50.

13. Kroenke K, Spitzer RL. The PHQ-9: a new depression measure. Psychiatr Ann. 2002;32:509-15.

14. Roy D, Talajic M, Dubuc M, et al. Atrial fibrillation and congestive heart failure. Curr Opin Cardiol. 2009;24:2934 .

15. Ziaei F, Zaman M, Rasoul D, et al. The prevalence of atrial fibrillation amongst heart failure patients increases with age. Int JCardiol. 2016;214:410-1.

16. Boriani G, GlotzerTV,Santini M, etal. Device-detected atrial fibrillation and riskfor stroke: an analysis of $>10,000$ patients from theSOSAFproject (StrokepreventiOn Strategies based on Atrial Fibrillation information from implanted devices). Eur HeartJ.2014;35:508-16.

17. Healey JS, Connolly SJ, Gold MR, et al. Subclinical atrial fibrillation and the risk of stroke. NEnglJ Med. 2012;366:120-9.

18. Podd SJ, Sugihara C, Furniss SS, Sulke N. Are implantable cardiac monitors the "gold standard" for atrial fibrillation detection? A prospective randomized trial comparing atrial fibrillation monitoring using implantable cardiac monitors and DDDRP permanent pacemakers in post atrial fibrillation ablation patients. Europace. 2015;18:1000-5.

19. Ponikowski P, Voors AA, Anker SD, et al. ESC guidelines for the diagnosis and treatment of acute and chronic heart failure. Eur Heart J. 2016;37:2129-200.

20. Varma N, Epstein AE, Irimpen A, Schweikert R, Love C, TRUST investigators. Efficay and safety of automatic remotemonitoring for implantablecardioverter-defibrillator follow-up. Circulation. 2010;122:325-32.

21. Crossley GH, Boyle A, Vitense H, Mead CY. The CONNECT (clinical evaluation of remote notification to reduce time to clinical descision) trial: the value of wireless remote monitoring with automatic clinician alerts. J Am Coll Cardiol. 2011;37:1181-9.

22. Boehmer JP, Hariharan R, Devecchi FG, et al. A Multisensor Algorithm Predicts Heart Failure Events in Patients With Implanted Devices. Jacc HeartFail. 2017;5:216-25. 